\title{
Mesures d'économie et risques liés à la responsabilité civile
}

\section{Eva Druey Just}

Dr iur., avocate à Coire

\author{
Suite à la présentation du catalogue de propositions d'un groupe d'experts du DFI, \\ les discussions sur les mesures visant à freiner les coûts de la santé se sont de nou- \\ veau intensifiées à l'automne dernier. Nombre de ces mesures présentent des \\ risques supplémentaires liés à la responsabilité civile - un point auquel la discus- \\ sion sur les objectifs d'économie a accordé trop peu d'importance jusqu'à présent.
}

\section{Les efforts pour maîtriser les coûts}

\section{Rationnement et rationalisation}

Depuis des années, tous les acteurs de la santé s'efforcent de réduire les coûts. Pour eux, il s'agit d'éviter l'inutile sans perte de qualité et d'organiser les processus de manière plus efficiente [1]. Récemment, un groupe d'experts interdisciplinaire du DFI a enrichi le débat en présentant une palette de nouvelles idées avec, pour certaines, des changements particulièrement importants à la clé, comme l'introduction de budgets globaux [2].

Si la rationalisation est tout à fait souhaitable, il ne faut pas la confondre avec le rationnement: ici, il ne s'agit pas simplement d'améliorer l'efficience mais aussi de limiter des prestations qui, du point de vue médical, seraient nécessaires avec, pour conséquence inévita-
La collecte d'idées visant à réduire les coûts de la santé bat son plein. De nombreuses mesures, dont une planification renforcée de l'offre, la réduction des prix des médicaments ou l'augmentation de la pression sur les prix, sont déjà en cours d'application. Des idées comme l'introduction de budgets globaux, le respect strict du principe de l'ambulatoire avant I'hospitalier ou le renforcement du contrôle des factures sont en discussion. Le fait que les mesures proposées augmentent les risques liés à la responsabilité civile des fournisseurs de prestations à bien des égards n'est toutefois guère évoqué dans cette discussion. Ces risques accrus ne seront pas sans conséquences: soit les mesures d'économie seront contournées, soit les primes des assurances responsabilité civile connaîtront une hausse significative. Dans les deux cas, cela signifie un pas en arrière sur la voie de la maîtrise des coûts. Les acteurs de la santé feraient bien de ne pas laisser complètement de côté le problème de la responsabilité civile dans la discussion sur les mesures d'économie. ble, une perte de la qualité. Le rationnement est, d'une part, en contradiction avec les objectifs de l'art. 43 al. 6 de la LAMal [3] qui exige que «les soins soient appropriés et leur qualité de haut niveau, tout en étant le plus avantageux possible». D’autre part, le médecin doit faire preuve de diligence et se conformer aux règles de l'art et doit ainsi des soins de qualité à son patient. Un rapport de tension s'établit donc entre la baisse des coûts et le devoir de qualité. Nous développons ci-dessous comment ce rapport peut impacter les risques liés à la responsabilité civile.

Mesures visant à freiner la hausse des coûts: ce qui est connu, ce qui est nouveau

\section{Les mesures connues}

Les mesures déjà mises en place au niveau politique [4] se concentrent sur une planification renforcée de l'offre, en particulier par la planification hospitalière et la limitation des admissions pour les cabinets médicaux. En parallèle, des réductions ont été décidées sur les prix des médicaments, ainsi que des interventions dans la structure tarifaire TARMED pour éviter les incitatifs négatifs et la sur-rémunération [5].

Les baisses des prix ont été atteintes moins par l'action politique que par la voie judiciaire. Les hôpitaux ont subi des baisses sensibles de l'indemnisation de leurs prestations ambulatoires. Mais aussi les prix de base des traitements hospitaliers ont tendance à être aujourd'hui plus bas qu'au moment de l'introduction des forfaits par cas en 2012 [6]. L'arrêt du Tribunal fédéral sur le myozyme illustre de manière spectaculaire les efforts judiciaires mis en œuvre pour réduire les coûts [7]. Dans les considérants de cet arrêt, le Tribunal fédé- 
ral lorgne sur la méthode intitulée "QALY», sans pour autant l'approuver ou la rejeter concrètement. D'après cette méthode, les coûts de traitement pour un patient pourraient être limités à une somme déterminée il était question d'une somme de CHF 100000 - par année de vie supplémentaire avec une qualité de vie élevée (quality-adjusted life year pour QALY).

Les mesures évoquées relèvent majoritairement du domaine de la rationalisation. Le rationnement, à savoir les limitations concrètes des prestations et de la qualité, apparaît seulement de manière isolée. La limitation de 20 minutes instaurée au $1^{\text {er }}$ janvier 2018 avec le TARMED 1.09 pour une consultation normale constitue au sens strict un rationnement mais accepte des exceptions pour les patients nécessitant plus de soins. De ce fait, elle ne devrait pas avoir d'impact médical négatif. Un rationnement caché apparaît en revanche lorsque les prix ont été tellement baissés qu'il n'est plus possible de fournir des soins en couvrant les frais malgré un haut niveau d'efficience. Le recours à la méthode QUALY qui, jusqu'à présent, n'a pas été mise en œuvre de manière cohérente au niveau politique ou judiciaire, déboucherait sur des rationnements très nets.

\section{Nouvelles mesures en discussion ou en planification}

Il n'est pas possible ici de se pencher sur l'ensemble des mesures en discussion ou en planification. Seules quelques mesures qui suscitent actuellement de vives discussions sont mentionnées ci-après [8]:

- Définition d'un plafond limitant l'augmentation des coûts à la charge de l'assurance obligatoire des soins (AOS): la hausse admise pour les dépenses de santé est fixée au préalable. Selon cette proposition formulée, les quatre grandes catégories de coûts à la charge de l'AOS (hôpitaux, médecins en cabinets privés, médicaments et autres) se verraient attribuer chacune un budget, soit un plafond assorti de sanctions en cas de dépassement. Lorsque le budget annuel est atteint, un traitement doit être repoussé jusqu'à la prochaine période budgétaire, si c'est médicalement indiqué. Les "prestations nécessaires» [9] doivent pouvoir continuer d'être fournies.

- L'ambulatoire avant l'hospitalier: les interventions simples, pour lesquelles un séjour hospitalier n'est pas nécessaire, doivent être obligatoirement exécutées en ambulatoire. Les cantons de Zurich et de Lucerne ont déjà édicté leurs listes respectives de ces interventions, la Confédération va leur emboîter le pas au $1^{\text {er }}$ janvier 2019 [10].

- Health Technology Assessment (HTA): il s'agit de vérifier le catalogue des prestations obligatoires et éventuellement d'exclure des prestations de l'AOS respectivement de ne les reprendre qu'après un examen strict de l'indication.

- Contrôle des factures: aujourd'hui déjà, les prestations médicales prises en charge par l'AOS doivent être efficaces, appropriées et économiques (critères EAE). Si un traitement déjà dispensé est considéré ultérieurement comme non conforme aux critères EAE, l'assurance refuse de le prendre en charge. Il est question de renforcer encore ce contrôle. Il deviendra nécessaire de demander plus souvent une garantie de prise en charge par l'assurance.

Les quatre mesures évoquées ici vont davantage dans le sens du rationnement que celles déjà mises en œuvre. En particulier les plafonnements globaux, mais aussi les autres mesures, impliquent que des prestations ne soient pas fournies malgré l'indication médicale. Les trois dernières mesures, en particulier, s'immiscent dans la compétence thérapeutique du médecin et transfèrent des décisions thérapeutiques concrètes aux assureurs-maladie, aux médecins-conseils ou encore au catalogue des prestations obligatoires.

\section{Maîtrise des coûts et risques liés à la res- ponsabilité civile}

\section{La diligence due par le médecin}

Tout médecin est tenu à la diligence dans l'exercice de son art. Il doit toujours «traiter son patient conformément aux règles de l'art afin de le protéger dans sa vie et sa santé. Il doit notamment observer la diligence requise par les circonstances et qu'on peut attendre de lui. En principe, le médecin répond de tout manquement à ses devoirs.» [11] Au cas par cas, ce sont les attentes légitimes du patient qui sont déterminantes [12]. Si la diligence exigée fait défaut, le médecin s'expose à des actions en dommages et intérêts ou à des conséquences pénales. La jurisprudence à ce sujet est inchangée depuis des années. Les réflexions sur les coûts lui sont en grande partie restées étrangères.

\section{La compatibilité des mesures d'économie avec le droit de la responsabilité civile}

Les mesures visant à réduire les coûts: un argument libératoire en cas de dommage

Les efforts de ces dernières années pour réduire les coûts n'ont guère pu influencer la jurisprudence du droit de la responsabilité civile pour la simple raison que cette dernière se joue entre le médecin et le patient. A l'inverse, les mesures d'économie concernent toujours le financement. Ce dernier, d'après notre système, n'impacte généralement pas le rapport entre le fournisseur de prestations et le patient mais celui du 
fournisseur de prestations avec l'assurance-maladie obligatoire.

Lorsque les mesures de réduction des coûts virent au rationnement, il est possible que les règles de l'art médical ne puissent plus être respectées. S’il en découle un cas de responsabilité civile, les médecins et les hôpitaux doivent essayer de s'en libérer en indiquant les mesures prescrites. Cela signifie qu'ils doivent reporter les obligations découlant de leur rapport juridique avec l'assurance-maladie sur leur relation juridique avec le patient. Il a été mentionné plus haut que les attentes légitimes du patient sont déterminantes pour la diligence due par le médecin. Pour libérer ce dernier, il faut donc que le patient connaisse les règles et adapte ses attentes en conséquence. Un patient ne pourra pas se plaindre qu'une opération ait été réalisée en ambulatoire lorsque celle-ci figure sur une liste cantonale qui interdit une hospitalisation. Il en va de même pour les traitements et les médications non prescrits s'ils ont été exclus des prestations obligatoires. Dans de tels cas, le devoir du médecin et/ou de l'hôpital sera d'informer le patient des règles applicables, dans le but de tempérer ses attentes légitimes.

\section{Les risques de la définition des priorités}

En revanche, il sera très difficile de libérer un médecin d'allégations concernant son manque de diligence dans la manière d'avoir prodigué un traitement, si la mesure d'économie concernée n'est pas suffisamment tangible pour être comprise comme une consigne pour un traitement concret. L'exemple de la liste des interventions ambulatoires restera l'exception qui confirme cette règle. En définitive, la plupart des mesures d'économie se traduisent simplement par des réductions budgétaires. Mais la décision des fournisseurs de prestations doit continuer d'avoir sa place dans le budget. En d'autres termes, ils doivent fixer les priorités: si les temps d'attente augmentent aux urgences en raison d'une enveloppe budgétaire réduite, le triage doit fonctionner encore mieux; si un budget global conduit à des temps d'attente pour des interventions choisies, il convient d'estimer les conséquences de cette attente; si une consultation ne peut durer que vingt minutes, il faut définir quel en est le contenu absolument nécessaire. Autrement dit, les réductions budgétaires donnent une toute nouvelle signification au devoir du médecin de fixer des priorités. Trier entre ce qui est nécessaire, souhaitable et inutile augmente incontestablement l'efficience mais est aussi susceptible d'occasionner des dommages. Et précisément dans le cas de plafonds généraux ou d'autres objectifs budgétaires, ce tri relève de la seule responsabilité des fournisseurs de prestations.

\section{Les risques du transfert de la responsabilité $d u$ traitement}

Les efforts d'économie cachent un risque supplémentaire lorsqu'une partie de la responsabilité du traitement est transférée à l'assurance-maladie, c'est nommément le cas avec la proposition de renforcer le contrôle des factures. Ce transfert des compétences décisionnelles est problématique du point de vue du droit de la responsabilité civile car le médecin doit certes remplir les objectifs de l'assureur mais il reste responsable du patient. Dans sa thèse, Brigitte Pfiffner Rauber, juge au Tribunal des assurances sociales de Zurich, part à juste titre du principe que le Parlement avait au moins prévu les mêmes responsabilités thérapeutiques pour les médecins-conseils que pour les médecins traitants: deux propositions de la minorité avaient été «rejetées en arguant que les normes de responsabilité civile et pénale en vigueur s'appliquent également aux médecins-conseils» [13]. Pourtant, en l'état actuel des choses, cette disposition est restée lettre morte. Au final, c'est le médecin, et ce sera toujours le médecin et non la caisse-maladie, qui doit un traitement médicalement correct à son patient. Conformément au droit en vigueur, une assurancemaladie a déjà la possibilité de refuser une garantie de prise en charge sans devoir sérieusement s'attendre à des prétentions en responsabilité civile. Si le médecin estime que le traitement refusé est nécessaire, il doit recommander au patient de le suivre mais à ses frais. En particulier lorsque le danger est aigu et qu'un financement par le patient n'est pas raisonnablement exigible, le médecin traitant doit choisir entre la peste et le choléra: si le traitement a lieu, la facture risque de rester impayée. Si le traitement n'a pas lieu, le patient risque d'exiger des prétentions en responsabilité civile pour omission de traitement approprié.

\section{Conclusion: n'oubliez pas la responsabilité civile!}

L'analyse des mesures, qu'elles soient déjà mises en œuvre, prévues ou encore en discussion, visant à freiner la hausse des coûts montre que pratiquement chacune d'elles peut potentiellement augmenter les risques liés à la responsabilité civile des fournisseurs de prestations. Plus les règles sont vagues, plus la responsabilité des médecins est importante lors de la hiérarchisation des prestations pouvant encore être fournies et plus le risque d'erreur de définition des priorités s'accroît. En devenant plus concrète, une règle a tendance à donner des arguments aux fournisseurs de prestations pour qu'ils se libèrent de leur responsabilité civile. Mais ces derniers ont l'obligation d'infor- 
mer leurs patients des rationnements. Des plafonds concrets ayant un impact sur les traitements présentent par ailleurs l'inconvénient de restreindre le médecin dans ses compétences de traitement.

Le débat sur les efforts d'économie doit toujours être mené en gardant à l'esprit le droit de la responsabilité civile. Sinon, les risques liés à la responsabilité civile augmenteront. Les fournisseurs de prestations y seront confrontés soit en combattant les mesures d'économie et peut-être en les contournant; ou alors, ils acceptent les risques accrus, ce qui devrait contribuer à une hausse significative des primes d'assurance responsabilité civile. Une telle augmentation constitue un nouveau facteur de coût. Par conséquent, négliger les risques de responsabilité civile au moment de fixer les mesures d'économie signifie toujours poser une entrave totale ou partielle aux objectifs d'économie.

\section{Conflits d'intérêt}

Dans le cadre de son activité d'avocate, Eva Druey Just conseille et représente plusieurs fournisseurs de prestations de santé.

\section{Références}

1 Groupe de travail interdisciplinaire et indépendant «Répartition équitable des ressources en santé publique»: Manifeste pour une répartition équitable des remèdes dans le domaine de la santé pu-

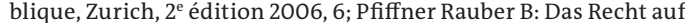
Krankheitsbehandlung und Pflege; Zum Behandlungsanspruch von Krankenversicherten im Rahmen der Wirtschaftlichkeit unter besonderer Berücksichtigung der Langzeitpflege, Forum Gesundheitsrecht Band 8, Zurich 2003, p. 221s.
2 Les idées réunies dans le rapport du 24.8.2017 du groupe d'experts du DFI «Mesures visant à freiner la hausse des coûts dans l'assurance obligatoire des soins" peuvent être consultées sur https://www.bag.admin.ch/bag/fr/home/themen/versicherungen/krankenversicherung/kostendaempfung-kv.html.

3 Le Conseil fédéral a écrit dans son message sur la LAMal en 1992 «Une telle option permet d'éviter tout rationnement des prestations, quantitatif ou qualitatif", FF 1992 I 77, 80 et 116.

$4 \mathrm{Cf}$. le groupe d'experts du DFI, $2^{\mathrm{e}}$ note de bas de page.

5 Cf. Office fédéral de la santé publique, fiche d'information "Adaptation du tarif médical TARMED» du 18.10.2017 et le communiqué de presse à ce sujet de l'OFSP du 16.8.2017.

6 Les baisses de rémunérations dans le TARMED sont également liées à l'intervention dans la structure tarifaire, cf. $5^{\mathrm{e}}$ note de bas de page. Exemples de baisse des coûts ambulatoires par le Tribunal administratif fédéral: arrêts du TAF C-4505/2013, C-2380/2012, C-1220/2012, C-3175/2013; la tendance à la baisse des prix de base du secteur hospitalier peut être consultée via la Conférence suisse des directrices et directeurs cantonaux de la santé, aperçu des listes hospitalières cantonales et des tarifs hospitaliers, état 10.8.2017, en ligne sur www.gdk-cds.ch.

7 ATF 136 V 392; à ce sujet par ex. Wasserfallen J-B/Junod V. Rationnement des soins: qu'implique l'arrêt «Myozyme»? BMS. 2011;45: 1751ss.

8 Rapport du groupe d'experts du DFI du 24.8.2017, $2^{\mathrm{e}}$ note de bas de page.

9 Cit. du rapport du groupe d'experts du DFI du 24.8.2017, $2^{\mathrm{e}}$ note de bas de page, p. 34.

10 Communiqué de presse commun de la Direction de la santé du canton de Zurich et du Département sanitaire et social du canton de Lucerne du 26.10.2017, en allemand, https://gd.zh.ch/internet/ gesundheitsdirektion/de/aktuell/medienmitteilungen.html; modification de l'ordonnance sur les prestations de l'assurance des soins (OPAS): RO 2018967 s.; d'autres cantons prévoient la même chose.

11 ATF 120 Ib 411 E. 4a.

12 Arrêt du Tribunal fédéral 4C.53/2000 consid. $1 \mathrm{~b}$.

13 Pfiffner Rauber B, note finale en haut, p. 148, note 50, avec mention du Bulletin officiel RS du 17.12.1992, p. 1324 (cahier des délibérations p. 218). 